\title{
A cellular model of swarm intelligence in bees and robots
}

\author{
Martina Szopek \\ Artificial Life Lab of the \\ Department of Zoology \\ Karl-Franzens University \\ Graz, Austria \\ martina.szopek@uni- \\ graz.at
}

\author{
Martin Stefanec \\ Artificial Life Lab of the \\ Department of Zoology \\ Karl-Franzens University \\ Graz, Austria \\ martin.stefanec@uni- \\ graz.at
}

\author{
Michael Bodi \\ Artificial Life Lab of the \\ Department of Zoology \\ Karl-Franzens University \\ Graz, Austria \\ michael.bodi@uni-graz.at
}

\begin{abstract}
Gerald Radspieler
Artificial Life Lab of the

Department of Zoology

Karl-Franzens University

Graz, Austria

gerald.radspieler@uni-

graz.at
\end{abstract}

\begin{abstract}
We present here a simple cellular model of random motion and social interaction of young honeybees making swarm intelligent decisions in complex dynamic temperature fields. We model also behaviors of stationary robots that affect those bees. Our study looks for a first as-simple-as-possible approach towards modeling such a bio-hybrid system. Our model predicts observed collective behaviors qualitatively very well by modeling a correlated random walk and a simple social interaction mechanism. We found that even a very simple 2 -dimensional cellular model with a limited state space of 16 bit per cell suffices. Ultimately, the simplicity of the model allows fast and distributed computation. This will allow us to search for interesting swarm intelligent robotic algorithms for creating novel bio-hybrid systems composed by real animals and autonomous rule-driven cellular robots by using stochastic optimization techniques.
\end{abstract}

\section{CCS Concepts}

-General and reference $\rightarrow$ General conference proceedings; •Computing methodologies $\rightarrow$ Multi-agent systems; Artificial life; Mobile agents; Agent / discrete models; Cooperation and coordination;

\section{Keywords}

honeybees, cellular model, collective decision making, biohybrid system, mixed society, robots

Corresponding author

Permission to make digital or hard copies of all or part of this work for personal or classroom use is granted without fee provided that copies are not made or distributed for profit or commercial advantage and that copies bear this notice and the full citation on the first page. To copy otherwise, to republish, to post on servers or to redistribute to lists, requires prior specific permission and/or a fee. BICT 2017, March 15-16, Hoboken, United States

ISBN 978-1-63190-148-5

DOI: 10.4108/eai.22-3-2017.152396

Copyright $\odot 2017$ EAI

\author{
Thomas Schmickl \\ Artificial Life Lab of the \\ Department of Zoology \\ Karl-Franzens University \\ Graz, Austria \\ thomas.schmickl@uni- \\ graz.at
}

\section{INTRODUCTION}

Temperature plays a crucial role in the collective physiology of a honeybee colony. It allows rapid brood growth and is thus a fundamental aspect for the population dynamics of a honeybee colony $[17,3]$. It was found that young honeybees show a complex behavior in heterogeneous temperature fields similar to the thermal conditions found in the brood nest area of honeybee colonies. For example, a group of young honeybees can locate itself at the optimal temperature spot among several options containing also local optima [25]. This is achieved without individually visiting and comparing all these places, thus it is achieved collectively as a result of swarm intelligence [5], [14]. Interestingly, it was found that these collective behaviors can be produced by a very simple set of individual rules, known as the agentbased BEECLUST algorithm [22], as it is depicted in Figure 1a. These rules are based on temperature-mediated resting behaviors that bees exhibit after bee-to-bee collisions. It allows the group (the 'swarm') to pick the optimum from multiple choices without explicit communication, without global self-localization of agents and without using explicit memory inside of the agents [15]. This algorithm has been implemented in robot swarms $[23,12]$ and agent-based models $[4,13]$. Its main advantage is that the agents themselves (e.g., through their placement in the environment) act as collective memory and communicate indirectly via modulating collision probabilities at various places in the habitat.

Research question: Here we aim to maximally simplify the BEECLUST model. We look for a cellular model [10], inspired by cellular automata [27], requiring a minimum number of bits (states) per cell, to achieve collective behaviors that resemble those observed in real honeybees. To validate such a rule set we compare it to observed honeybee behaviors in several different sets of environmental conditions.

This is an important endeavor in the project ASSISIbf [2], which associates an array of locally interacting spatially fixed 'robots' to allow interaction with the bees (Figure 1b). In ASSISIbf, these non-moving robots are called CASUs (Combined Actuator and Sensor Units). Although they are immobile they exhibit autonomous agency, e.g. by sensing, 


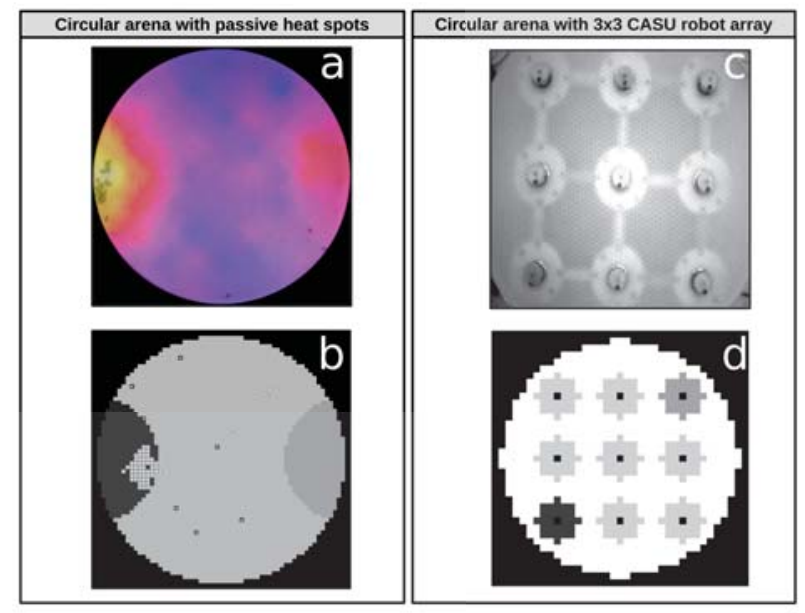

Figure 2: Experimental setups with bees and their representation in the cellular model. (a) Circular arena with two passive heat spots on each side produced by ceramic heat lamps. (b) Representation of this setting in our model. (c) Circular arena containing a 3x3 CASU robot array. (d) Representation of this array setting in our cellular model. (b and d) Black colored patches: Walls/obstacles. Grey patches: Empty fields, shades of grey indicate local temperature: The darker the color is, the colder it is there. Black boxes with white ' $\mathrm{X}$ ': running bees. Grey boxes with white ' $\mathrm{X}$ ': resting bees. (d) Each CASU robot is represented as an obstacle surrounded by a local heat spot (see also Figure 8).

by computing and by actuating. The robots sense nearby bees with infrared sensors and they perform estimates of local bee numbers or densities [21]. Besides sharing these data with local neighbors they can also act on bees by locally producing temperature or by cooling down. This is known to affect the behaviors of bees, as it is already expressed in the BEECLUST algorithm (Figure 1a). In fact, an array of CASUs can act as a heat-modulating cellular automaton that interacts with an embedded swarm of real bees (Figure 1bc).

\subsection{The model}

Our model is a 2-dimensional cellular model. Its basic structure is a lattice or array $C$ consisting of $N_{x}$ by $N_{y}$ cells that represent possible locations of honeybees in an experimental arena. We assume that each cell can hold one bee, thus each cell represents an area of approx. $1.0 \mathrm{~cm}^{2}$. We assume that each bee can move by one cell length every time step. Assuming the speed of an average bee at $1.0 \mathrm{~cm} / \mathrm{sec}$, this shows that our model uses a time resolution of $\Delta t=1.0$ sec. One run in our model was lasting for 1800 or 5400 time steps, what corresponds to 30 or $90 \mathrm{~min}$. For our model validation we emulated the following experiments conducted with real bees: Observations of aggregation behaviors in flat temperature fields of various temperatures and aggregation at a global optimum with and without local optima (Figure $2 \mathrm{ab})$. We varied population sizes of bees in most of those experiments. Later, we also emulated a $3 \times 3$ CASU array in a circular arena (Figure 2cd). The configuration of each cell $i$ at each time step $t$ is expressed by the following cell states:

- $\operatorname{wall}(i) \in\{$ False,True $\}$ indicates whether or not this cell represents a wall or an obstacle.

- bee $(t, i) \in\{$ False,True $\}$ indicates whether or not this cell holds a bee.

- $\operatorname{orientation}(t, i) \in\{N, N W, W, S W, S, S E, E, N E\}$ indicates the orientation of a bee at cell $i$.

- $\operatorname{spot}(i) \in\{$ False,True $\}$ indicates whether or not this cell is a designated heat spot area.

- $\tau_{\text {wait }}(t, i) \in\{0,1, \ldots, 30,31\}$ indicates the waiting time of a bee according to the locally sensed temperature.

- temperature $(t, i) \in\{28.0,28.5, \ldots, 37.5,38.0\}$ indicates the local temperature at cell $i$ at time step $t$. Thus we assume a very coarse temperature sensing of bees that is able to discriminate temperatures in $\Delta$ temperature $=$ $0.5^{\circ} \mathrm{C}$ steps. This is a conservative assumption, several studies indicated the ability to discriminate differences down to $\Delta$ temperature $=0.25^{\circ} \mathrm{C}$, e.g., see [7].

In total the model requires 5 bit to encode the temperature information, 5 bit to encode the waiting time information and 6 bit to encode the local presences and orientation of bees, walls and heat spots. This yields a total amount of information of 16 bit per cell as cell state space. However, in most of our experiments we use only two or three different temperatures. This further reduces the required amount of info per cell to 13 bit per cell. Two of these binary states do not change over time (wall(i) and spot(i)), further decreasing the required state space to 11 bits per cell for dynamic data. Several pieces of info $\left(\tau_{\text {wait }}(t, i)\right.$, temperature $(t, i)$ and orientation $(t, i)$ ) are not evaluated for every cell but only for those cells that actually hold bees, thus the relevant total state space further reduces to the following equation 1

total_state_space $(t)=8 \mathrm{bit} \cdot N_{x} \cdot N_{y}+8 \mathrm{bit} \cdot N_{\text {bees }}$.

\subsection{The initialization of the cellular automa-} ton model

- Set for each cell $i$ in $C$ : bee $(0, i)=$ False,wall $(i)=$ False, temperature $(0, i)=$ AmbientTemp, $\tau_{\text {wait }}(t, i)=$ $0, \operatorname{spot}(i)=$ False.

- Then set for all cells $j$ which fulfill the boundary conditions of our experiment: $\operatorname{wall}(j)=$ True.

- Then set for all cells $k$ which represent heat spot areas: $\operatorname{spot}(k)=$ True.

- Then select those cells $m$ that belong to each temperature spot $(\operatorname{spot}(i)==$ True $)$ and set there: temperature $(0, m)=$ CorrespondingSpotTemp.

- Select randomly (uniform probability distribution) a set of $N_{\text {bees }}$ cells $(l)$ with $($ bee $(0, l)==$ False) and $(\operatorname{wall}(l)==$ False $)$ and $(\operatorname{spot}(l)==$ False $)$ and set there: $\operatorname{bee}(0, l)=$ True. The sampling is done without replacement, the number of chosen cells $N_{\text {bees }}$ corresponds to the number of bees used in experiments with real bees.

\subsection{The honeybee behavior model}

Figure 3 shows the flow of logic in our cellular model in a python-like pseudo code. The basic idea is that we select all cells (line (l.) 01) and check whether or not they contain a bee ( $l$. 02). If a cell contains a bee we check if the bee is in resting state $\left(\tau_{\text {wait }}(t, i)>0, l\right.$. 03). In this case we will just reduce the waiting counter $\tau_{\text {wait }}(t, i)$ by a value of 1 ( $l$. $04)$. Otherwise, if $\tau_{\text {wait }}(t, i)$ is already zero, we will try to move the bee to a new cell $(l$. 05$)$.

Next, we determine, through drawing a random (uniform) 

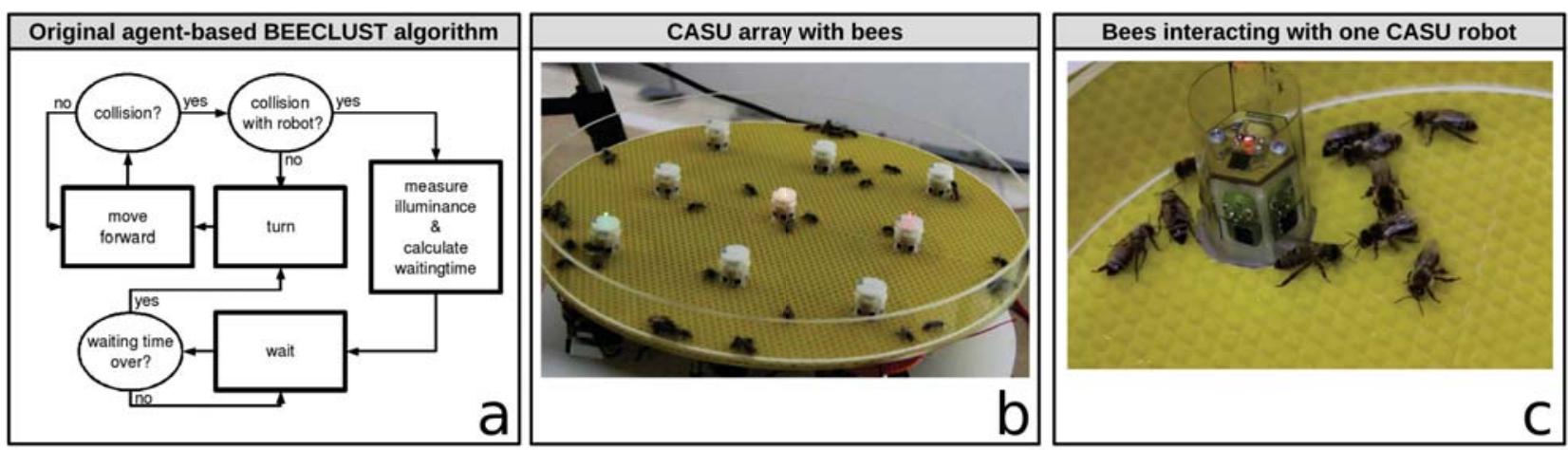

Figure 1: (a) The agent-based BEECLUST algorithm that is to be represented by a set of cellular model rules. (b) Array of CASU robots with bees moving between them. (c) A group of bees aggregating around one CASU robot because it warms up the local environment above the ambient room temperature.

number between 0.0 and 1.0 and a probability threshold $\alpha$, whether the bee will continue to move in its current direction or pick a new direction randomly $(l$. $06-09)$. In the first case we select the cell to which orientation $(t, i)$ points from the 8 neighbor cells as new TARGET (l. 10). In the second case, we select a random cell from the 8 neighboring cells as TARGET and adjust the value of orientation $(t, i)$ accordingly ( $l .08)$. The higher the value of $\alpha$, the more likely the bee will choose always a newly randomized direction. Thus, with $\alpha=1.0$ the bee will perform an uncorrelated random walk (like brownian motion) and with $\alpha=0.0$ the bee will always move in a straight line until it bumps into a wall $(l$. 12 - 15) or into another bee(l. 16).

Then we randomly select $\sigma$ other cells from the remaining 7 neighborhood cells $(l$. 11). At these cells and also at the TARGET cell we check whether or not another bee is there (l. 16), assuming that a bee can detect the local presence of other bees not only when directly bumping into it head-on, but also based on other random body contacts, e.g. at its antennae or feet.

If one of the selected neighborhood cells (including the cell TARGET) contains another bee, the focal bee switches to the resting (waiting) mode. The duration of the resting is dependent on the local temperature (temperature $(t, i)$ ) based on a stimulus-response function: We set $\tau_{\text {wait }}(t, i)=$ $f($ temperature $(t, i))(l .17)$. From empirical data we know that bees do not wait after meeting another bee in cold areas and that they wait longer with increasing temperatures up to $36^{\circ} \mathrm{C}-38^{\circ} \mathrm{C}$. Thus we modeled the $f()$ according to equation 2 as

$$
f\left(T^{*}\right)= \begin{cases}\tau_{\text {wait }}^{\text {min }} & \text { if } T^{*}<T_{\text {min }}^{*} \\ \tau_{\text {wait }}^{\text {max }} & \text { if } T^{*}>T_{\text {max }}^{*} \\ \tau_{\text {wait }}^{\text {min }}+T^{*} \cdot \frac{\left(\tau_{\text {wait }}^{\text {max }}-\tau_{\text {wait }}^{\text {min }}\right)}{\left(T_{\text {max }}^{*}-T_{\text {min }}^{*}\right)} & \text { else }\end{cases}
$$

which floors the waiting time at $\tau_{\min }$ below $T_{\min }^{*}$, ceils it at $\tau_{\text {wait }}^{\max }$ above $T_{\max }^{*}$ and scales it linearly increasing from $\tau_{\text {wait }}^{\min }$ to $\tau_{\text {wait }}^{\max }$ between $T_{\min }^{*}$ and $T_{\max }^{*}$ according to the local temperature $T^{*}$. This partially linearized stimulus-response function roughly mimics the sigmoid function described in $([15],[18])$

Finally, in case our bee did not detect another bee, it will

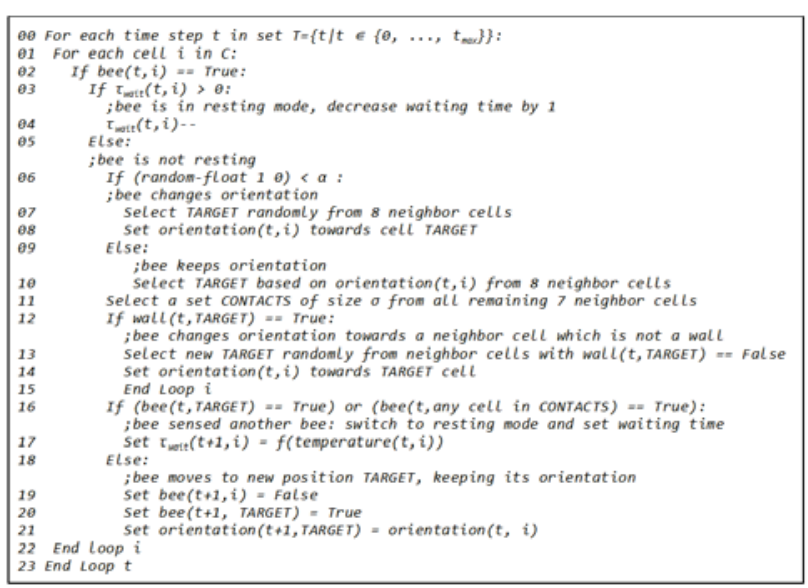

Figure 3: Pseudo-code of our model's core control logic.

move to the selected TARGET cell by setting bee $(t, i)=$ False (l. 19) and bee $(t+1$, TARGET) $=$ True $(l .20)$ and orientation $(t+1$, TARGET $)=\operatorname{orientation}(t, i)(l$. 21).

\subsection{Experimental procedures}

In order to validate our model against empirical data, we simulated 5 types of experiments that were conducted with real bees in a circular arena:

In those experiments we used ceramic heat lamps to heat selected areas of an arena to desired temperatures. We kept those temperatures stable with PID controllers and floor sensors [26]. We parameterized our model accordingly to those conditions and ran a set of experiments to gather corresponding model predictions.

Then we perform a model analysis especially focusing on our free parameters $\sigma$ (social parameter) and $\alpha$ (correlatedness of the random-walk). This analysis checks for model sensitivity to our free parameters and informs us about the effect of those parameters on predicted collective behaviors.

Finally, we perform one simulation experiment in which we simulate an array of 9 CASU robots organized in a $3 \times 3$ array that perform a pre-programmed change of their temperature production profile and predict the corresponding 
behaviors of a group of bees in such a CASU robot array.

In all our simulations we used a specific set of default parameter values (see Table 1). In most runs we used only those parameter values. In specific parameter sweeps we varied one of those parameters systematically.

\section{EXPERIMENTS AND THEIR RESULTS}

\subsection{Model validation}

For model validation against empirical data we conducted the following sets of simulation runs with our model:

Experiment 1: Two group sizes $\left(N_{\text {bees }}=24\right.$ and $N_{\text {bees }}$ $=128$ ) run in a homogeneous temperature field of $29{ }^{\circ} \mathrm{C}$ $(N=30$ repetitions/group size, runtime $=1800$ steps $)$. Our cellular model predicts the emergence of almost no clusters for 24 bees and the emergence of a few small clusters for 128 bees in this environment after 1800 time steps, corresponding to 30 min with real bees. All these clusters emerge (randomly) distributed across the arena.

See Figure 4a-d for a comparison of typical real bee results and typical model results. Concerning the ratio of lonely bees to bees in small (2-3 bees per cluster) and in large clusters $(4+$ bees per cluster) our model predicts a similar qualitative distribution of cluster sizes compared to the observations with real bees in the same environment (Figure $5 \mathrm{a}$, left two columns).

Experiment 2: Two group sizes $\left(N_{\text {bees }}=24\right.$ and $N_{\text {bees }}=$ $128)$ run in a homogeneous temperature field of $36{ }^{\circ} \mathrm{C}(N=$ 30 repetitions/group size, runtime $=1800$ steps $)$. Model predictions show a high degree of similarity compared to empirical data, see Figure 4e-h: With 24 bees only few large clusters emerge while with 128 bees several large clusters emerge at random places across the arena. The numerical comparison of the ratio of lonely bees to bees in small and in large clusters shows a high agreement to the empirical observed data with real bees (Figure $5 \mathrm{a}$, right two columns).

Experiment 3: A group of bees $\left(N_{\text {bees }}=64\right)$ runs in a temperature gradient field produced by one heat spot of $36^{\circ} \mathrm{C}$ on the left side of the arena with an ambient temperature (pessimum) of $31^{\circ} \mathrm{C}(N=30$ repetitions, runtime $=$ 5400 steps). Our model predicts the emergence of large clusters preferentially in the warm left zone in this environmental configuration (see Figure 5b), what is in accordance to empirically observed data.

Experiment 4: Five group sizes of bees $\left(N_{\text {bees }} \in 1,6,24\right.$, $64,128)$ run in a complex temperature field of two overlapping gradients produced by two differently warm heat spots $\left(36^{\circ} \mathrm{C}\right.$ left spot, $31^{\circ} \mathrm{C}$ pessimum, $32^{\circ} \mathrm{C}$ right spot; $N=30$ repetitions/group size, runtime $=5400$ steps). Figure 6 shows a strong accordance of our simulation to observations of real bees: In both cases the agents prefer to aggregate on the left side (global optimum) and there is also slight aggregation on the right side (local optimum). With increasing numbers of bees, the aggregation of the bees first grows stronger on the left side, until the group size of $N_{\text {bees }}$ $=128$ bees is reached. At this group size the local optimum on the right side starts to attract bees to the cost of the left side aggregation.

Figure $5 \mathrm{c}$ shows that the time budget of single bees in all 3 zones closely resembles the Uniform random Distribution Model (UDM). Contrary, in runs with groups of bees the fraction of bees across those 3 zones strongly deviates from this pure random-choice model prediction. This suggests that the observed distributions of bees in groups are nonrandom, agreeing with findings in real bees [25].

\subsection{Analysis}

A preliminary version of our model contained only an uncorrelated random walk (like brownian motion) where agents didn't have any directionality and also interacted only if a bee selected an already occupied cell as a new movement target. This preliminary model failed to be validated as it showed qualitatively similar behavior compared to bees but all dynamics of collective choices were happening very slow (more than one order of magnitude slower than in the real honeybee system). Thus we introduced a correlated walk (controlled by the parameter $\alpha$ ) and a stronger local social interaction (controlled by the parameter $\sigma$ ) to our model.

The original model is still a special case of our final model presented here, just with $\alpha=1.0$ and $\sigma=0$. In order to analyze the importance and effects of these two free parameters we conducted the following parameter sweeps:

Experiment 5: Systematic parameter sweep of the social parameter $\sigma$ from 0 to 7 with $N_{\text {bees }} \in\{6,24,64,128\}$ bees in the complex temperature field (see experiment 4 ). $N=100$ repetitions/step/group size, runtime $=5400$ steps.

Figure 7 a shows that the parameter $\sigma$ has a huge effect on aggregation behavior and collective choice: The higher the number of bees is, the sharper this effect appears: values of $3<=\sigma<=4$ yield the highest aggregation success after 5400 time steps, while with higher bee populations a value of $\sigma=2$ is more efficient. With higher population densities it becomes more likely to trigger a bee-to-bee contact in our model, thus a lower $\sigma$ value suffices.

Experiment 6: Systematic parameter sweep of the correlatedness of the random-walk $(\alpha \in\{0.00,0.05, \ldots, 1.00\}$ with $N_{\text {bees }}=64$ simulated bees in complex temperature fields (see experiment 4). $N=100$ repetitions/step, runtime $=5400$ steps.

Figure 7b shows that also the parameter $\alpha$ has a significant effect on collective decision making: A value of $\alpha=$ 0.1 , which is making an agent change its direction every 10 time steps on average, yields the sharpest collective decision making. This value allows the agent to cross the arena in short time but still prevents it from getting stuck at the wall, like it is the case with $\alpha=0.0$. Higher values of $\alpha$ decrease choice quality, as the diffusion of agents across the arena is slowed down and thus collective discrimination of both sides gets more and more slowed-down or even impaired.

\subsection{First test application with emulating a pre- programmed CASU robot array}

Experiment 7: In this experiment we used an array of $3 \times 3$ CASU robots to 'guide' the bees along a path: One of the CASU robots is always presenting a global optimum temperature $\left(36^{\circ} \mathrm{C}\right)$ while another CASU robot is always presenting a local optimum temperature $\left(32^{\circ} \mathrm{C}\right)$ to test the bees' collective discrimination ability for moving aggregation targets: The two optima move across the array, as after some time the CASU robots that produce heat cease their activities and neighboring CASU robots pick up their work. This way we generate a dynamic environment in which heat spots change over time.

Figure 8 shows, that the bee aggregations predicted by our model closely resemble the collective behaviors observed in real bees in such an experiment. 
Table 1: Default parameter settings.

\begin{tabular}{|l|l|l|l|}
\hline Parameter/Constant & Value(s) & Unit & Source/Reasoning \\
\hline $\mathrm{N}_{x}, \mathrm{~N}_{y}$ & 60,60 & {$[$ cells $]$} & Dimension of our real world experimental arena \\
\hline$N_{\text {bees }}$ & 64 & {$[\mathrm{bees}]$} & Standard setting also in real bee experimentation \\
\hline AmbientTemp & 31 & {$\left[{ }^{\circ} \mathrm{C}\right]$} & Standard setting also in real bee experimentation \\
\hline$\alpha$ & 0.1 & dimensionless & Free parameter \\
\hline$\sigma$ & 3 & {$[$ cells $]$} & Free parameter \\
\hline$t_{\max }$ & 5400 & {$[$ time steps $]$} & Represents 90 minutes of real time \\
\hline$T_{\min }^{*}, T_{\max }^{*}$ & 29,37 & {$\left[{ }^{\circ} \mathrm{C}\right]$} & Lower and upper limit of simplified waiting time curve \\
\hline$\tau_{\text {wait }}^{\text {min }}, \tau_{\text {wait }}^{\text {max }}$ & 0,19 & {$[$ time steps $]$} & Minimum and maximum waiting time \\
\hline
\end{tabular}
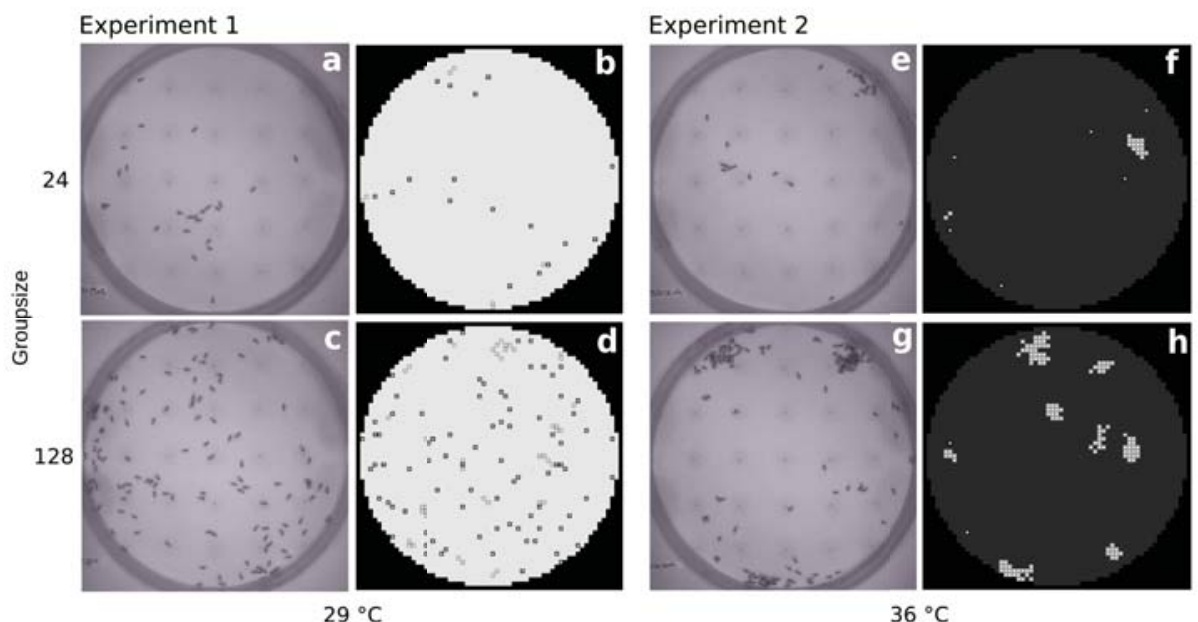

$36^{\circ} \mathrm{C}$

Figure 4: Exemplary snapshots of the final distribution of bees in honeybee experiments $(N=10$ repetitions/group size, runtime $=30 \mathrm{~min})$ and simulated bees $(N=30$ repetitions/group size, runtime $=1800$ steps $)$ in flat temperature fields. Experiment 1 (a)-(d): 24 and 128 bees at $29^{\circ} \mathrm{C}$; Experiment $2(\mathrm{e})-(\mathrm{h}): 24$ and 128 bees at $29^{\circ} \mathrm{C}$.
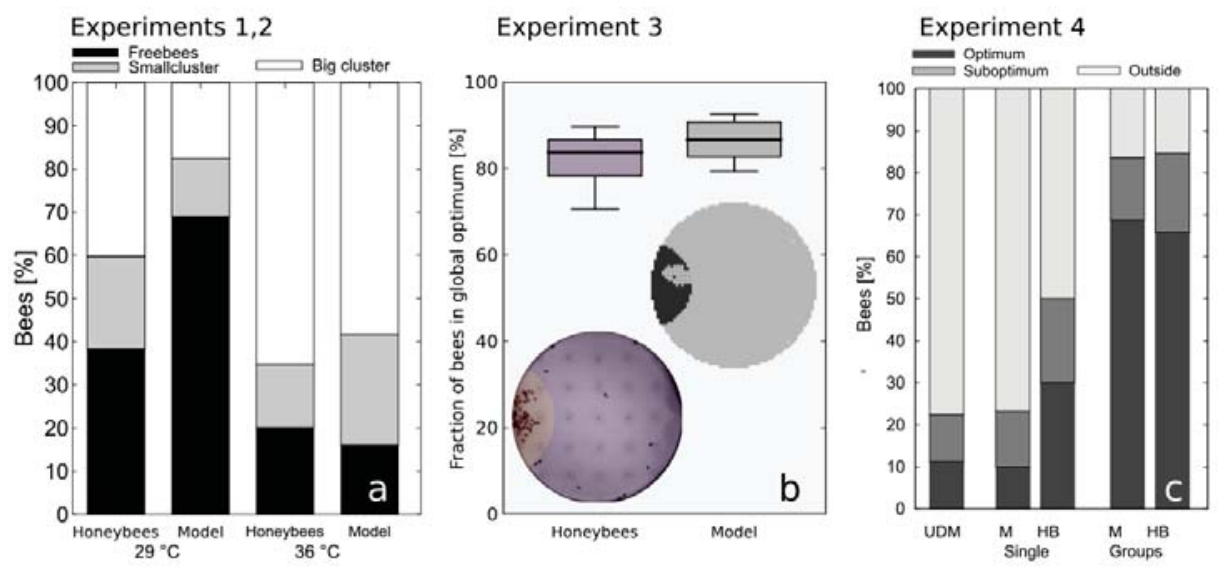

Figure 5: (a) Cluster analysis: Percentage of free bees, bees in small clusters (2-3 bees/cluster) and bees in big clusters $(4+$ bees/cluster) with real honeybees and with simulated bees at the end of the experiments (30 min and 1800 time steps) for homogeneous temperatures of $29^{\circ} \mathrm{C}$ (Experiment 1) and $36^{\circ} \mathrm{C}$ (Experiment 2). (b) Percentage of bees in the global optimum $\left(36^{\circ} \mathrm{C}\right)$ after $30 \mathrm{~min}$ for real bee experiments and simulated bees (Experiment 3). (c) Comparison of the results of Experiment 1 to the UDM (Uniform random Distribution Model) and empiric data with real honeybees. Graph shows the median percentage of honeybees (HB) and modeled bees $(\bar{M})$ in the three different zones (global optimum, local optimum (suboptimum), outside the optima (pessimum)) at the end of the experiments for single bees and groups of bees (all group sizes accumulated) compared to the UDM. 


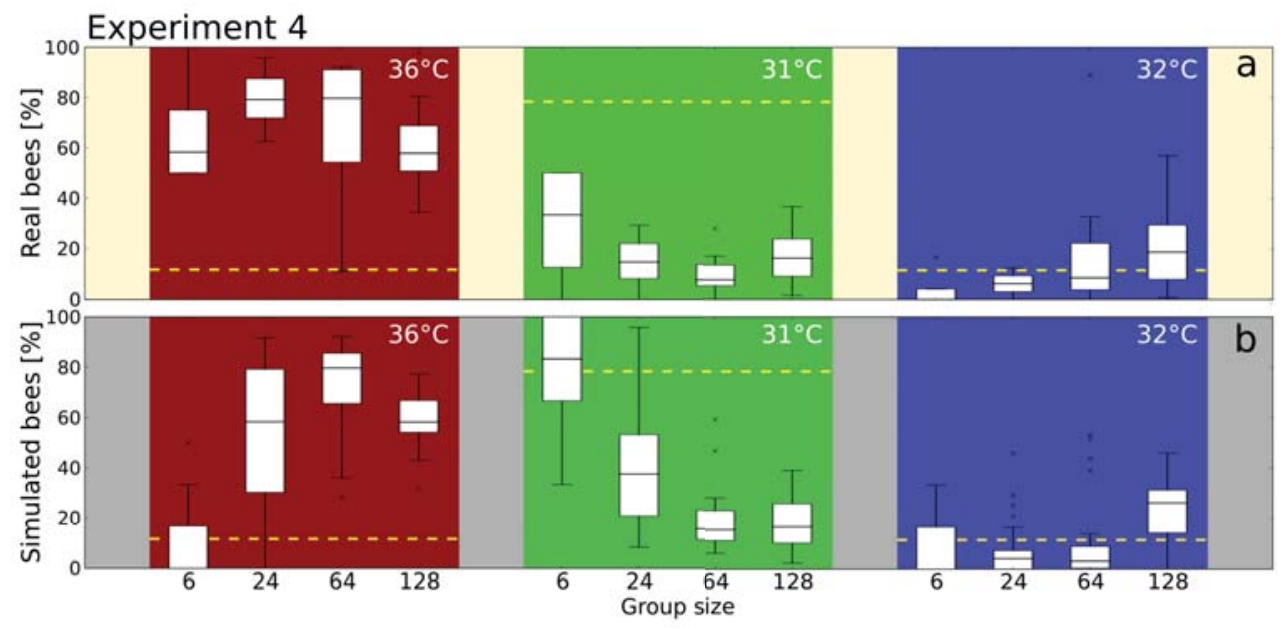

Figure 6: Median percentage of bees in the three different temperature zones (Red: global optimum at $36^{\circ} \mathrm{C}$; Blue: local optimum (suboptimum) at $32^{\circ} \mathrm{C}$; Green:outside of these optima (pessimum) at $31^{\circ} \mathrm{C}$ for (a) experiments with real honeybees $(N=8$ repetitions/group size) and (b) simulated bees $(N=30$ repetitions/group size) for all group sizes $(6,24,64,128$ bees $)$. The dashed line indicates the predicted values by the UDM for these zones. Deviations from this line indicate non-random collective behavior.
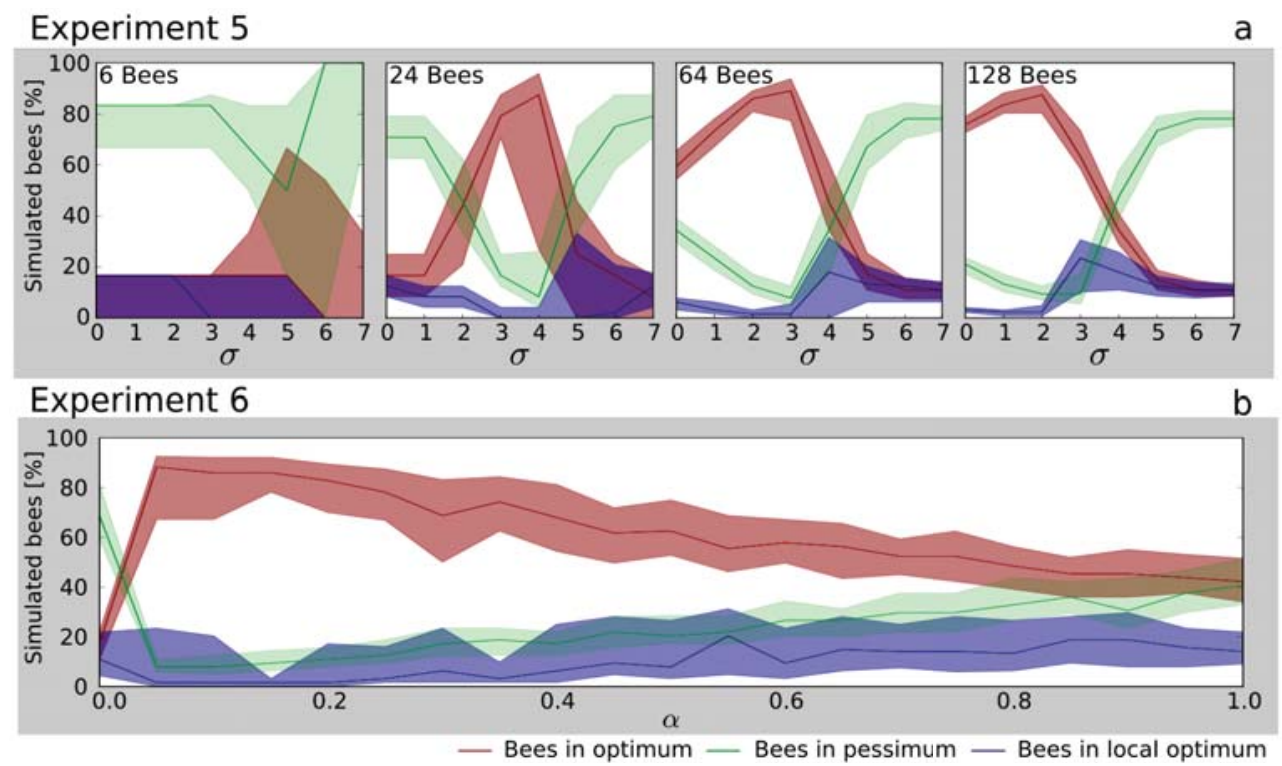

Figure 7: Systematic sweeps of $\alpha$ and $\sigma$ in a temperature field with a global optimum $\left(36^{\circ} \mathrm{C}\right)$, a pessimum $\left(31^{\circ} \mathrm{C}\right)$ and a local optimum $\left(32^{\circ} \mathrm{C}\right.$ ) (see also Experiment 4). (a) Sweep of the social parameter $\sigma$ from 0 to 7 with different group sizes (6, 24, 64 and 128 simulated bees). Each panel shows a connected interpolated boxplot: Median (solid lines) and IQR (filled areas) of 30 repetitions after 5400 time steps. (b) Sweep of the random-walk correlation parameter $\alpha$ from 0.0 to 1.0 in 0.05 wide steps with 64 simulated bees. Connected interpolated boxplot: Median (solid lines) and IQR (filled areas) of 30 repetitions after 5400 time steps. Red: bees in optimum, green: bees in pessimum, blue: bees in local optimum 

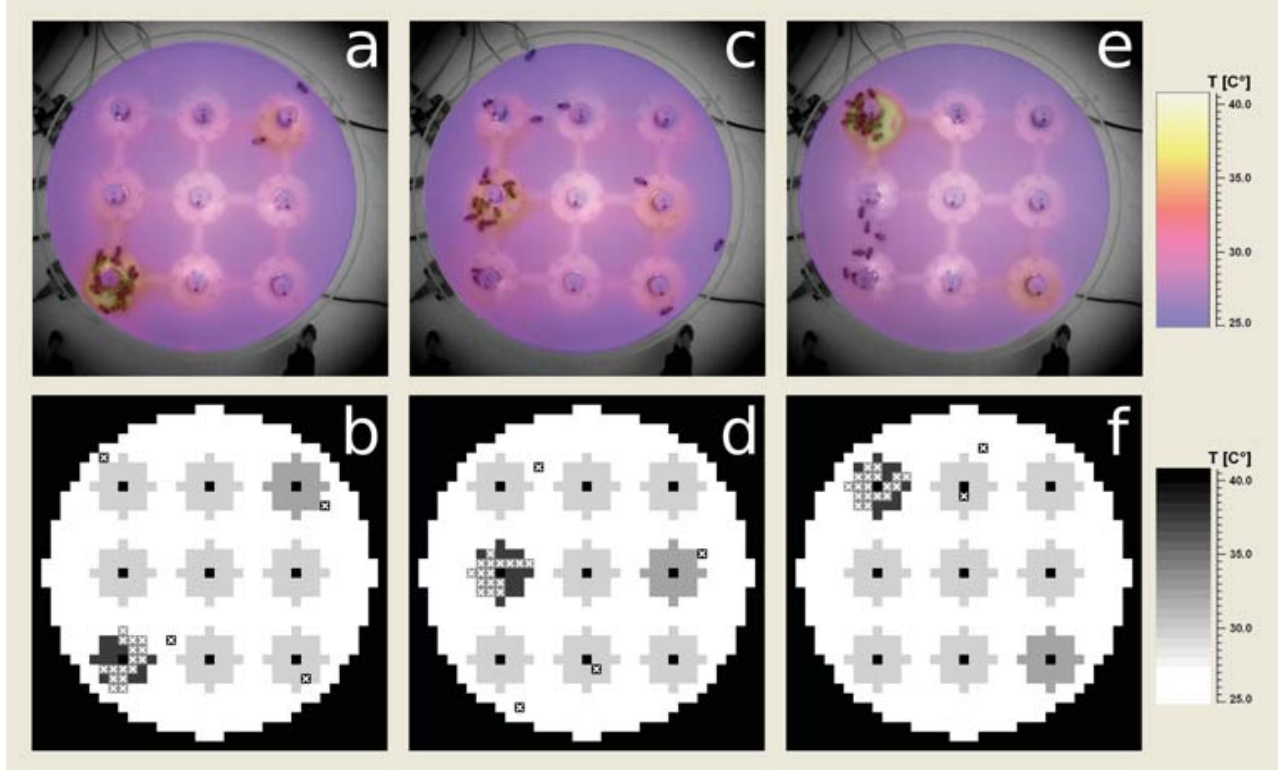

Figure 8: Snapshots of the $3 \times 3$ CASU robot array set with robots actively heating at different time steps and their representation in our cellular model. The behavior of CASUs at runtime is: One CASU always produces a global optimum at $36^{\circ} \mathrm{C}$ while another CASU produces a local optimum at $32^{\circ} \mathrm{C}$. All 7 other CASUs heat to $29^{\circ} \mathrm{C}$, producing additional local optima. Temperatures are indicated by a color map overlay in (a),(c) and (e) and by a grey-scale map in (b), (d) and (f). (a) Screenshot after 15 minutes of runtime, global optimum: bottom left CASU, local optimum: top right CASU. (b) Model representation of CASU array setting of (a). (c) Screenshot after 30 minutes of runtime, global optimum: mid left CASU, local optimum: mid right CASU. (d) Representation of CASU array setting of (c). (e) Screenshot after 45 minutes of runtime, global optimum: top left CASU, local optimum: bottom right CASU. (f) Representation of CASU robot array setting of (e).

\section{DISCUSSION \& CONCLUSION}

Our model captures collective behaviors of honeybees by closely resembling observations of real honeybees in comparable situations. All major aspects of emergent collective behaviors are captured by our simple cellular model.

We first validated our model against empirical data collected with honeybees with two population sizes and two homogeneous temperature settings (Figures 4, 5a). Then we validated the model against empirical data with heterogeneous environments with one or two temperature spots (global \& local optima, Figures 5b,c, 6). Then we analyzed the importance of the two free parameters $\alpha$ and $\sigma$, showing a strong density dependence of $\sigma$ (social interaction). The collective discrimination ability is sensitive to $\alpha$, showing a decrease in choice quality with higher values (Figure 7). Finally, we demonstrated the potential of the model for predicting environments that are dynamic, e.g., by being changed through autonomous robots that socialize with the animals (Figure 8). In this setting we could also demonstrate (with real bees and with our cellular model) that in a setting of one global optimum and 8 local optima with differing quality, the BEECLUST behavior is still capable to select the global optimum.

For the sake of simplicity, we abstracted away many features of the real system: We assumed that all honeybees move by a correlated random walk, where the parameter $\alpha$ models the correlatedness of this random walk [6]. In fact it was shown that honeybees tend to keep their direction for some time (inertia, correlated random walk [11]). Several other motion strategies are reported to exist for moving young honeybees, like 'wall followers', 'sitters', 'goal seekers' [11]. We purposely restricted ourselves from going into such level of detail in our model building to find the simplest possible model that can describe the main features observed in the system. Also the environmental model was kept as simple as possible: For example, we assumed that heat spots are having sharp edges, building 'plateaus' in the temperature fields instead of appearing as smooth temperature gradients.

In addition, we showed here a first approach to model autonomous robots that modulate bee behavior by heating or cooling. Later, we will also incorporate these robots' sensing capabilities and their autonomous agency into the model.

Similar representations of animal behaviors have been used in [24] to model older honeybees in self-organized temperature clusters. In contrast to our work, which models young honeybees, the model of [24] was focused on a full-hive context and on older bees that produce heat by themselves. Thus it was more complex, for example, a diffusion-andinsulation model of heat flows was included there. Simple animal interaction models following a cellular approach were also used to model food-transportation in ant trails [1] and the water storage and exchange in paper wasps [9].

The simplicity of the rules and the high-speed computation, that results from this model simplicity, will in future allow us to conduct exhaustive searches for individual behaviors that lead to interesting swarm-level behaviors by stochastic optimization methods, e.g., machine learning [19], genetic programming [16] or evolutionary computation [8], [20]. By doing so, we can allow the robotic nodes in the system to perform learning at run-time, what will allow them 
to integrate themselves smoothly into the honeybee society and then to act as 'probes within the swarm' for us to further investigate this swarm intelligent honeybee system. The model presented here is a crucial ingredient in this scientific plan, as it is computationally slim, allowing exhaustive searches of the parameter space. In addition, it is very open to extensions and elaborations to satisfy future demands.

\section{AUTHOR CONTRIBUTIONS}

M.Sz. made exp. 1,2,4, 7 and analysis (analysis \& graphs). M.St. simulated exp. 5-7 and made analysis and graphs and extended the initial model. M.B. made exp. 7 and made Fig. 1, 2 and a literature survey. G.R. exp. 3 and its analysis. T.S. had the initial idea, designed the study and programmed the initial cellular model. All authors contributed at approx. equal extent to the writing of the article.

\section{ACKNOWLEDGEMENTS}

Supported by EU FET project ASSISIbf, \#601074; Austrian Science Fund (FWF) grant P23943-N13 (Rebodiment).

\section{REFERENCES}

[1] C. Anderson, J. Boomsma, and J. Bartholdi. Task partitioning in insect societies: bucket brigades. Insectes Sociaux, 49:171-180, 2002.

[2] ASSISIbf. Project website, 2014 http://http://assisi-project.eu/.

[3] F. Bodenheimer. Studies in animal populations. ii. seasonal population-trends of the honey-bee. The Quarterly Review of Biology, 12(4):406-425, 1937.

[4] M. Bodi, R. Thenius, M. Szopek, T. Schmickl, and K. Crailsheim. Interaction of robot swarms using the honeybee-inspired control algorithm beeclust. Mathematical and Computer Modelling of Dynamical Systems, 18(1):87-100, 2012.

[5] E. Bonabeau, M. Dorigo, and G. Theraulaz. Swarm Intelligence: From Natural to Artificial Systems. Oxford Univ. Press, 1999.

[6] E. A. Codling, M. J. Plank, and S. Benhamou. Random walk models in biology. Journal of The Royal Society Interface, 5(25):813-834, 2008.

[7] H. Heran. Untersuchungen über den Temperatursinn der Honigbiene (Apis mellifica) unter besonderer Berücksichtigung der Wahrnehmung strahlender Wärme. Journal of Comparative Physiology A: Neuroethology, Sensory, Neural, and Behavioral Physiology, 34:179-206, 1952.

[8] J. H. Holland. Adaptation in Natural and Artificial Systems. MIT Press, Cambridge, MA, USA, 1992.

[9] I. Karsai and A. Runciman. The effectiveness of the "common stomach" in the regulation of behavior of the swarm. In MATHMOD 2009 - 6th Vienna International Conference on Mathematical Modelling, pages 851-857. ARGESIM Publishing House, 2009.

[10] S. A. Kauffman. Emergent properties in random complex automata. Physica D: Nonlinear Phenomena, 10(1):145 - 156, 1984.

[11] D. Kengyel, H. Hamann, P. Zahadat, G. Radspieler, F. Wotawa, and T. Schmickl. Potential of heterogeneity in collective behaviors: A case study on heterogeneous swarms. In Principles and Practice of Multi-Agent Systems, PRIMA 15, page 1, 2015.

[12] D. Kengyel, T. Schmickl, H. Hamann, R. Thenius, and K. Crailsheim. Embodiment of honeybee's thermotaxis in a mobile robot swarm. In 10th European Conference on Artificial Life (ECAL'09), volume $5777 / 5778$ of $L N C S$. Springer-Verlag, 2011.
[13] D. Kengyel, R. Thenius, K. Crailsheim, and T. Schmickl. Influence of a social gradient on a swarm of agents controlled by the BEECLUST algorithm. Advances in Artificial Life, Proceedings of the 12th European Conference on the Synthesis and Simulation of Living Systems, ECAL13, 12:1041-1048, 2013.

[14] J. Kennedy and R. C. Eberhart. Swarm Intelligence. Morgan Kaufmann, 2001.

[15] S. Kernbach, R. Thenius, O. Kornienko, and T. Schmickl. Re-embodiment of honeybee aggregation behavior in an artificial micro-robotic swarm. Adaptive Behavior, 17:237-259, 2009.

[16] J. Koza. Genetic Programming: On the Programming of Computers by Means of Natural Selection. MIT Press, 1992.

[17] F. Kronenberg and H. C. Heller. Colonial thermoregulation in honey bees (apis mellifera). Journal of Comparative Physiology B: Biochemical, Systemic, and Environmental Physiology, 148(1):65-76, 1982.

[18] R. Mills, P. Zahadat, F. Silva, D. Miklić, P. Mariano, T. Schmickl, and L. Correia. Coordination of collective behaviours in spatially separated agents. In Advances in Artificial Life, 12th European Conference on Artificial Life (ECAL 2015), pages 579 - 586. MIT Press, 2015.

[19] M. Mitchell, P. T. Hraber, and J. P. Crutchfield. Revisiting the edge of chaos: Evolving cellular automata to perform computations. Complex Systems, 7(2):89-130, 1993

[20] I. Rechenberg. Evolutionsstrategie. Optimierung technischer Systeme nach Prinzipien der biologischen Evolution. Frommann Holzboog, 1973.

[21] Z. Salem and T. Schmickl. The efficiency of the rules-4 classification learning algorithm in predicting the density of agents. Cogent Engineering, 1(1):986262, 2014.

[22] T. Schmickl and H. Hamann. BEECLUST: A swarm algorithm derived from honeybees. In Y. Xiao, editor, Bio-inspired Computing and Communication Networks. CRC Press, March 2011.

[23] T. Schmickl, R. Thenius, C. Möslinger, G. Radspieler, S. Kernbach, and K. Crailsheim. Get in touch: Cooperative decision making based on robot-to-robot collisions. Autonomous Agents and Multi-Agent Systems, 18(1):133-155, February 2008.

[24] D. J. T. Sumpter and D. S. Broomhead. Shape and dynamics of thermoregulating honey bee clusters. Journal of theoretical Biology, 204:1-14, 2000.

[25] M. Szopek, T. Schmickl, R. Thenius, G. Radspieler, and K. Crailsheim. Dynamics of collective decision making of honeybees in complex temperature fields. PLOS ONE, 8(10):e76250, 102013.

[26] G. R. Williams, V. Dietemann, J. D. Ellis, and P. Neumann. An update on the coloss network and the 'beebook: standard methodologies for apis mellifera research'. Journal of Apicultural Research, 51(2):151-153, 2012.

[27] S. Wolfram et al. Theory and applications of cellular automata, volume 1. World scientific Singapore, 1986. 